\title{
La violence endémique en Afrique
}

Endemic violence in Africa

\section{Séverin Cécile Abéga}

\section{CpenEdition}

\section{Journals}

Édition électronique

URL : http://journals.openedition.org/apad/199

DOI : 10.4000/apad.199

ISSN : 1950-6929

Éditeur

LIT Verlag

Édition imprimée

Date de publication : 1 juin 2003

Référence électronique

Séverin Cécile Abéga, "La violence endémique en Afrique », Bulletin de l'APAD [En ligne], 25 | 2003, mis en ligne le 08 mars 2006, consulté le 21 septembre 2020. URL : http://journals.openedition.org/apad/ 199 ; DOI : https://doi.org/10.4000/apad.199

Ce document a été généré automatiquement le 21 septembre 2020

Bulletin de l'APAD 


\title{
La violence endémique en Afrique
}

\author{
Endemic violence in Africa
}

\author{
Séverin Cécile Abéga
}

1 Les études sur la violence se multiplient, avec parfois des ambitions très larges. Les explications du phénomène sont variées. Lee (2000) la rattache au degré de cohésion à l'intérieur d'une communauté, Roscoe (1996), aux bénéfices qu'elle procure et à la personne humaine. Newbury (1998) quant à lui lie par exemple le génocide rwandais à l'ethnicité, aux intérêts matériels, aux conditions écologiques et au genre. A côté de ceux qui veulent tout expliquer, ou trouver une explication unique ${ }^{1}$ à un phénomène aussi multiforme qu'universel, nous proposons des approches basées sur des travaux de terrain. Comment comprendre en effet la violence? Comme le prolongement, l'intensification du conflit, ou son contraire (Wieviorka, 2004:20)? Cependant, le problème est aussi celui de la perpétuation. Qu'est-ce qui la maintient ou la répète ? La violence devient en effet endémique ; elle se répète, se perpétue, mais aussi se répand, envahissant le pays, traversant les frontières, embrassant plusieurs couches sociales. L'endémicité est donc pour nous une notion liée au temps et à l'espace. Le conflit est au centre de la structure. Il est présent dans toutes les sociétés, et celles-ci en tiennent compte en le jouant ou en le provoquant à travers une théâtralisation ou des rituels. Ces manifestations permettent ainsi de le prévenir ou de le régler. Le deuil, le mariage et d'autres rites de passage, qui sont des moments de reformulation sociale, donnent ainsi lieu à une intense théâtralisation du conflit.

2 Le conflit survient quand s'écartent des intérêts dans le même champ. Il a été analysé selon deux orientations : l'une, conservatrice; l'autre, plus transformatrice. Dans les sociétés africaines, il se manifeste souvent sous forme d'accusations en sorcellerie, et nous retrouvons cet aspect parmi les articles proposés ici. Pour Mary Douglas (1966), lorsque certaines catégories sociales situées dans les interstices hors structures menacent de disloquer celle-ci, elles sont accusées de sorcellerie, ce qui permet de les sanctionner et de régler par ce biais la menace. Pour Evans-Pritchard (1937), le conflit entraîne une transformation. Il donne à la structure un mouvement d'expansion et de contraction. Il maintient les lignes de démarcation, et les interventions destinées au règlement qui empêchent la dislocation définitive. Il ravive donc les principes 
structurants. Par sa manifestation, il indique les points de survenue des changements et entraîne ces derniers, ce qui permet à la structure de se transformer pour se maintenir. Le conflit amène donc une restauration de l'ordre ancien et un maintien de celui-ci. Le conflit ne suppose donc pas forcément la violence, même s'il peut l'entraîner. Il semble destiné à la prévenir. Il est structurant par le fait même qu'il est pris en charge par la structure. On est donc dans une situation où le vaccin peut transmettre la maladie. Pourquoi ce paradoxe?

3 Un élément dont la présence semble entraîner un processus de violence, et que permet d'identifier la synthèse de Nagengast (1994), est la force. Toutes les définitions de la violence, et toutes les formes de violence s'accordent sur l'usage de la force comme nécessaire à la manifestation de celle-ci. La violence, parce qu'elle introduit la force dans le conflit, est un début de destruction de l'autre parce que la force permet de minimiser ses intérêts. Il serait cependant dangereux de s'enfermer dans cette vision unilatérale parce qu'à l'inverse, une bouffée de violence peut aussi permettre une institutionnalisation de l'opposition, du fait qu'elle oblige à prendre en compte des divergences qu'on semblait ignorer. Mais souvent dans ce cas, il s'agit d'une réponse à l'usage d'une force qui nie l'un des centres d'intérêts. A cet égard, on peut dire que la violence a contribué à créer le sujet.

4 Violence physique ou violence morale, la nature de l'une permet de définir celle de l'autre. La force semble se manifester quand le conflit ne se développe pas entièrement, par exemple par l'existence d'un verrouillage qui empêche le conflit de s'exprimer. Cependant, je ne pense pas que la seule force suffise. En effet, la force est aussi présente dans la structure dans la mesure où le pouvoir est un élément structurel, et que le pouvoir se décompose en force et en légitimité. L'organisation interne de chaque groupe lui permet de mobiliser, si cela s'avère nécessaire, les moyens de contrôler les intrusions de la violence. Il y a ainsi une violence utile et une mauvaise violence, ce qui nous ramène à l'approche wébérienne, mais qui était aussi celle de Protagoras ou de Hobbes. La bonne violence permet la vie en groupe organisée, et en confiant le monopole à la structure, contribue à pérenniser celle-ci. Pendant longtemps, on a considéré, et on continue de le faire dans beaucoup de sociétés, que le père de famille avait le droit de battre ses enfants, et aujourd'hui encore, ce sont surtout les excès d'un époux qui frappe trop souvent ou trop brutalement sa femme qui sont réprimés. La famille, le lignage, le clan, la chefferie ou l'Etat reconnaissent donc à certaines personnalités, de par leurs fonctions et de par leur place au sein de la structure, un droit d'exercice de la violence. Mais justement, la lutte engagée contre ces formes pourtant reconnues jusque là comme légitimes montre bien l'ambivalence du phénomène, ambivalence dont les clientes des centres de santé de Niamey et de Bamako qu'étudient Hadiza Moussa et Aïssa Diarra expérimentent trop souvent le mauvais côté dans leur confrontation avec les sages-femmes.

5 La plupart des analyses offertes dans ce bulletin peuvent se regrouper sous le thème du déficit de l'Etat. Les brigandages organisés dans les zones frontalières entre le Cameroun, le Tchad et la Centrafrique peuvent aussi être interprétés comme une remise en question de l'Etat, et Claude Abé ne se gène pas ici de le faire, de même que la violence dans les centres de santé nigériens et maliens, telle que présentée par Hadiza Moussa et Aïssa Diarra. Les fonctionnaires de la santé, profitant de leur position dans le système pour sécréter des pouvoirs qui prennent tant d'ampleur qu'ils menacent de désarticuler le système par un trop grand élargissement des interstices, montrent bien 
les limites du fonctionnement des administrations étatiques : la faiblesse des systèmes de contrôle. Cet Etat devient lui-même bien relatif en Afrique centrale devant les multiples ingérences étrangères.

6 Cependant, expliquer la violence par l'affaiblissement de l'Etat n'est qu'un simple constat qui ne nous donne pas une vue suffisante du phénomène. Cette approche mécaniste reste trop générale et n'éclaire que la périphérie du phénomène tant qu'on n'apprend pas comment cet affaiblissement s'est enclenché. Surtout, nous ne sommes pas renseignés sur la perpétuation qui nous intéresse ici, mais sur une cause qui n'est manifestement pas à la source du phénomène. Il est possible de resserrer l'objectif par l'introduction des acteurs. On peut en effet en apprendre plus en nous situant à une échelle où les jeux de stratégie deviennent visibles.

7 Un auteur comme Malinowski (1941) nous met sur la voie lorsqu'il remarque que la violence engendre des profits. Cette position se retrouve dans les travaux de beaucoup d'auteurs (Kanazawa et al., 2000 ; Lee, 2000 ; Roscoe, 1996 ; Newbury, 1998). Ces profits peuvent être politiques ou économiques, collectifs ou individuels. La notion de bénéfice nous met cependant au cœur du problème dans la mesure où elle donne l'occasion de cerner l'acteur en mouvement. On traverse alors le territoire de la fonction pour celui du calcul, et ce qu'on calcule ainsi, ce sont les coûts, les pertes et les bénéfices. L'existence des bénéfices est en effet synonyme de stratégies pour les acquérir. Malinowski montre qu'un accès de colère ne peut expliquer une violence récurrente, impliquant tout un groupe. De même la violence reste-t-elle limitée dans les sociétés dont le système économique ne permet pas l'accumulation. Par contre, lorsque apparaît une possibilité d'accumulation, la violence peut s'étendre et se répéter à cause de l'augmentation de sa productivité car à ce moment là, elle produit des bénéfices.

8 Ce qui est intéressant, c'est ce rappel, capital à mon sens, surtout s'agissant d'une question aussi délicate que la violence, qu'aucun motif individuel ne peut expliquer un phénomène social. Mais au-delà, l'analyse de Malinowski permet de lier la violence aux stratégies des acteurs. Dans cette perspective, un système s'organise fait de jeux et de calculs pour jouir des avantages qu'offrent ces situations. Ces bénéfices peuvent s'évaluer en termes matériels, symboliques ou statutaires. Dans le cas des ingérences armées en Afrique centrale, les bénéfices sont à la fois économiques et politiques. L'exploitation des ressources du sous-sol, le trafic des armes, d'autres commerces lucratifs, les pillages de même que la conquête de positions favorables ou la limitation d'une influence rivale exercent un attrait suffisant pour mobiliser les armées et leur faire franchir la frontière des Etats au motif de secourir un allié ou de sécuriser ses propres frontières.

9 Le système qui organise le régicide chez les Tikar du Cameroun, par exemple, est vigilant sur le choix et le rôle des notables impliqués, et est si complexe que parce qu'il empêche les corps constitués impliqués dans le meurtre du roi de tirer profit de la violence. La perte de la légitimité transforme en effet le pouvoir en une force incontrôlée, dangereuse. Aussi est-il nécessaire de la restaurer périodiquement, quitte à infliger la violence à un individu pour en préserver le groupe.

Le jeu des calculs est plus net dans le travail de Vincent Dississa sur les funérailles au Congo Brazzaville. Qu'il s'agisse des voyous ou des yankés, on se rend compte que la violence est organisée par des groupes qui en tirent des bénéfices matériels et statutaires et pour cela ont tout intérêt à la voir se maintenir. Le même modèle s'applique aussi bien aux coupeurs de route camerounais qu'aux milices des guerres du 
Congo. On peut effectivement parler de la constitution d'une classe "d'entrepreneurs" de la violence. Celle-ci devient donc en quelque sorte une espèce d'industrie dans laquelle investissent certaines catégories. Mais comme dans toute industrie, l'investisseur n'est souvent pas l'ouvrier.

11 Cela ressort bien dans le complot qui aboutit à l'assassinat de la vieille Labou dans l'article de Vincent Dississa au cours d'une cérémonie funéraire. Si cette femme a été identifiée comme une sorcière dont l'élimination est souhaitée par certains membres de son lignage et notamment, les sœurs de celui dont on célèbre les obsèques, ce ne sont cependant pas celles-ci qui exécutent le meurtre. L'assassinat est commis par des jeunes venus de la ville, et connus pour leur penchant pour la violence. Ils reçoivent d'ailleurs un paiement pour ce crime. Ce schéma ressort aussi en ce qui concerne les miliciens congolais. Ils sont recrutés et armés par un groupe qui se tient derrière eux et se garde bien de s'engager physiquement dans la guerre. L'usage de la drogue organise la dépendance et surtout la dépersonnalisation de ces subalternes, ce qui les rend malléables et en fait des automates entre des mains expertes. L'usage de la drogue n'est donc pas un élément secondaire dans ces guerres. Car derrière le consommateur se tapit un fournisseur et se ramifient des réseaux. Claude Abé subodore aussi une pareille structuration dans les coulisses du banditisme transfrontalier des zargina. La classe de ceux qui tirent profit de la violence se divise donc en plusieurs strates, certaines exerçant sur un plan surtout local, d'autres se déployant sur plusieurs pays, voire plusieurs continents.

On retrouve là un élément déjà identifié plus haut, celui de l'existence d'acteurs internationaux, et qui montre la complexité des phénomènes de violence. La présence de ces entrepreneurs indique bien que les idéologies proclamées ne sont donc parfois qu'une simple façade, ce qui apparaît aussi dans les centres de planification familiale de Niamey étudié par H. Moussa, où le comportement violent des sages-femmes à l'égard de leurs patientes est justifié par le non paiement des salaires, même si ceux-ci sont versés régulièrement depuis plusieurs mois. Ici aussi, on voit bien que la sage-femme n'est pas seule à profiter de la violence qu'elle déploie dans son lieu de service, puisqu'il s'est créé un marché de restauration autour des centres de santé, marché qui en rappelle un autre, organisé autour des ministères à Yaoundé, où l'on mange et boit souvent aux frais des usagers des services publics désireux de s'attirer les bonnes grâces du fonctionnaire chargé de leur dossier.

13 Les analyses présentées ici, en centrant l'attention sur les acteurs de la violence, nous permettent donc de saisir au moins un des mécanismes qui rendent la violence endémique en Afrique par sa répétition, sa récurrence, sa contagion. Cet élément, c'est la constitution d'une classe d'entrepreneurs de la violence, doublée parfois d'une main d'œuvre chargée de l'exécution des hautes œuvres.

Il reste toutefois un dernier élément sans lequel la mécanique de la violence perd sa logique: c'est l'expulsion. Celle-ci est peut-être consciente et manifeste, ou inconsciente et implicite. Elle est souvent exprimée dans les métaphores. La métaphore sert cependant de support au stéréotype, au préjugé. On cesse d'identifier des individus pour nommer des catégories. Ainsi naissent le sauvage, la vermine, le gibier de potence. Les métaphores puisent dans le vocabulaire de la maladie (peste, lèpre, gangrène, virus), de l'animalité (divers noms d'animaux ou d'insectes: fauve, hyène, chacal, chien, porc, scorpion, pou, vermine), de la tératologie (monstre, hydre). La violence se justifie alors comme une obligation de nettoyage, association dont plus d'un auteur 
(Abega, 1987; Fontette, 1976; Héritier, 1999) a remarqué la familiarité dans le vocabulaire des nazis, de la radio des Mille collines ou dans le système de castes hindou, en référence à certains groupes sociaux. L'association la plus récurrente au mot purification est d'ailleurs venue, à la fin du millénaire dernier, du tribalisme européen, inspirateur des guerres des Balkans, et qui l'a accolé à l'adjectif ethnique.

La violence est consécutive à un processus de création de l'autre, processus dont on distingue bien les modalités chez les anciens Maka (Abega, 1997), qui divisent le monde en trois cercles : le lignage, ceux avec lesquels s'organisent des échangent de femmes, et ceux qu'on peut manger. On identifie ici l'autre dans ses deux modalités : partenaire ct ennemi. Celui qui est la cible privilégiée de la violence est exclu du cercle de l'entre-soi et de celui des partenaires, car on ne peut s'attaquer à ceux auxquels on s'identifie ou à ses partenaires sans courir le risque d'une autodestruction. La transformation métaphorique du chef en oryctérope permet d'en faire un bouc émissaire chez les Rukuba (Muller, 1992). Quand le chef tikar dort, ses sujets disent de lui : neto ne dela, «le chef est une antilope ». C'est dans cette posture que les notables chargés du meurtre royal viennent l'étrangler, avec la complicité de son épouse. L'expulsion se fait donc par l'extraction de la catégorie du soi et des partenaires, et un enfermement dans la deuxième catégorie de l'autre par une représentation d'une altérité dangereuse, nuisible ou méprisable. Cette expulsion est nécessaire pour briser l'identification et sortir ainsi du schéma du suicide. C'est celle de l'envoûteur à Brazzaville et ailleurs, mais aussi celle de la femme venue consulter à Niamey pour maitriser sa fécondité, et dont la sage-femme proclame à haute voix qu'elle est atteinte d'une MST.

Les entrepreneurs de la violence peuvent d'ailleurs eux-mêmes se retirer des cercles de l'identité et du partenariat quand ils attaquent la nuit en se masquant le visage en ce qui concerne les zargina, ou lorsqu'ils se droguent, pouvant alors tuer avec l'impression que c'est quelqu'un d'autre qui donne la mort à leur place, et anesthésiant par ce processus tout ce qui est émotion. Wiervorka, s'inspirant de Touraine, rattache ce processus au sujet. On a vu plus haut que la violence créait le sujet. Mais à l'inverse, quand le sujet ne peut se construire, la violence rôde. Le sujet se construit dans la relation à l'autre. Par ses effets euphorisants, le dédoublement de personnalité et la coupure avec la réalité ambiante qu'elle provoque, le recours à la drogue, par exemple, atténue et brouille ce lien. Le processus de stigmatisation et d'exclusion est donc lié à la perpétration, autant que la constitution d'une classe d'entrepreneurs annonce la perpétuation. Où est le lien?

Je pense que si le processus d'expulsion sert d'amorce à la violence, il est de l'intérêt des entrepreneurs d'enclencher ou d'intensifier celle-ci pour créer une situation propice à leurs intérêts. De là à présenter l'Afrique comme la victime d'un vaste complot international, thèse trop facile et malheureusement adoptée par beaucoup d'africains. Toutes les manifestations de la violence n'ont pas la même origine et ne sont pas liées entre elles. J'identifierai la permanence d'un double langage qui masque l'intérêt derrière le discours idéologique, moralisant ou provocateur, et rend difficile la recherche des solutions. 


\section{BIBLIOGRAPHIE}

Abéga S.C., 1987, L'esana chez les Beti, Yaoundé, Clé.

Abéga S.C., 1997, "L'évolution de la situation matrimoniale des femmes et des règles matrimoniales chez les Maka de l'Est Cameroun", Cahier de l'UCAC, 1 : 95-117.

Evans-Pritchard E.E., 1937, The Nuer, London, Oxford University Press, Traduction française, 1968, Les Nuer, Paris, Gallimard.

Fontette F. (de), 1976, Le racisme, Paris, PUF, Que sais-je?

Héritier F., 1998, De la violence, tome 2, Paris, Odile Jacob.

Kazawa S., Stll M.C., 2000, "Why Men Commit Crimes (And Why They Desist)", Sociological Theory, vol. $8, n^{\circ} 3: 434-447$.

Lee M.R., 2000, "Community Cohesion and Violent Predatory Victimization : A Theoritical Extension and Cross-National Test of Opportunity Theory", Social Forces, vol. 79. n² : 683-706.

Malinowski B., 1941, "An Anthropological analysis of War", The American Journal of Sociology, vol. $46, \mathrm{n}^{\circ} 4: 521-550$.

Muller J.C, 1991, "Entre mythe et réalité. ou pourquoi le chef rukuba est un oryctérope", L'Homme, 118, XXXI. $n^{\circ} 2: 67-78$.

Nagengast C., 1994, "Violence, Terror. and the Crisis of the State", Annual Review of Anthropology, vol. 23 (1994) : 109-136.

Newbury D., 1998, “Understanding Genocide”, African Sudies Review, vol. 41, n 1 : 73-97.

Roscoe P.B., 1996, "War and Society in Sepik New Guinea", The Journal of the Royal Antrhopological Institute, vol.2, $\mathrm{n}^{\circ} 4: 645-666$.

Wieviorka M., 2004, La violence, Paris, Balland.

\section{NOTES}

1.Les titres de ces travaux en affichent d'ailleurs clairement l'ambition : Why Men Commit Crimes (Kazawa et al, 2000), Understanding Genocide (Newbury, 1998), La violence, (Wieviorka, 2004), etc.

\section{AUTEUR}

\section{SÉVERIN CÉCILE ABÉGA}

Professeur d'anthropologie. MINREST/UCAC Yaoundé Cameroun ; sabega@yahoo.fr 